01

\title{
Стационарные и самоподобные волны в стержне с разномодульной нелинейностью, диссипацией и дисперсией
}

\author{
(C) В.Е. Назаров, С.Б. Кияшко \\ Федеральный исследовательский центр Институт прикладной фризики РАН, \\ 603950 Нижний Новгород, Россия \\ e-mail: v.e.nazarov@appl.sci-nnov.ru
}

Поступило в Редакцию 5 марта 2019 г.

В окончательной редакции 6 мая 2019 г.

Принято к публикации 29 июня 2019 г.

Исследовано распространение продольных упругих волн в стержне с разномодульной нелинейностью, линейной диссипацией и геометрической дисперсией фазовой скорости. Получены аналитические и численные решения для профилей стационарных волн и самоподобных периодических волн и импульсных возмущений, распространяющихся в таком стержне без изменения формы; проведен их графический анализ.

Ключевые слова: разномодульная нелинейность, диссипация, дисперсия, упругие волны.

DOI: $10.21883 /$ JTF.2020.01.48654.82-19

\section{Введение}

Существует довольно широкий класс твердотельных сред, обладающих разномодульными упругими свойствами, т.е. различными модулями упругости при сжатии и растяжении [1]. К таким средам относятся некоторые полимеры, композиционные и конструкционные материалы, грунты, а также материалы, содержащие трещины. Изучению нелинейного распространения продольных упругих волн в разномодульных (или бимодульных) средах посвящено большое число работ [1-12]. В этих работах рассматривалось как распространение волн в идеальной разномодульной среде, так и влияние диссипации и релаксации на эволюцию волн в таких средах. В разномодульных средах нелинейный режим распространения имеет место только для разнополярных волн, однополярные же возмущения распространяются линейно, с постоянными, но различными скоростями, зависящими от их полярности. Нелинейное искажение первоначально гармонической (разнополярной) волны в идеальной разномодульной среде происходит таким образом, что на каждом периоде в ее профиле, уже на сколь угодно малом расстоянии от излучателя, образуется „перехлест“ (неоднозначность), устраняемый введением искусственного разрыва - ударного фронта [5]. В результате такого искажения амплитуды высших гармоник нелинейной волны пропорциональны первой степени амплитуды первичной волны, при этом волна затухает до нуля на конечном расстоянии [5]. Линейные диссипация и дисперсия - всегда [7-12], а релаксация - только для относительно низкочастотных волн [8-10], предотвращают образование неоднозначностей в профиле волны в разномодульной среде и ее быстрое затухание (до нуля), однако амплитудные закономерности для высших гармоник волны сохраняются.
Изучение нелинейных волновых процессов (НВП) в разномодульных средах представляет научный и практический интерес. Научный интерес связан с выявлением закономерностей НВП в средах с неаналитической нелинейностью, а практический - с созданием эффективных методов диагностики их структуры. В частности, интерес к задачам о распространении продольных упругих волн в разномодульных средах во многом связан с линейной зависимостью разномодульной нелинейности от амплитуды деформации, что позволяет получать точные аналитические решения нелинейных волновых уравнений для таких сред.

В настоящей работе проводится теоретическое исследование распространения продольных упругих волн в стержне с разномодульной нелинейностью с учетом линейной диссипации и геометрической дисперсии фазовой скорости. Рассматривается распространение стационарных и автомодельных (самоподобных) разнополярных периодических волн и импульсных возмущений, распространяющихся в таком стержне без изменения формы.

\section{1. Основные уравнения}

Уравнение состояния разномодульного твердого тела имеет вид $[1,5,7]$ :

$$
\sigma(\varepsilon)=\left\{\begin{array}{ll}
E_{1} \varepsilon, & \varepsilon \geq 0 \\
E_{2} \varepsilon, & \varepsilon \leq 0
\end{array} \mid=E[\varepsilon-\gamma|\varepsilon|]\right.
$$

где $\sigma, \varepsilon-$ продольные напряжение и деформация, $E_{1,2}$ - модули упругости среды при ее растяжении и сжатии (для стержня это модули Юнга),

$$
E=\frac{E_{1}+E_{2}}{2}, \quad \gamma=\frac{E_{2}-E_{1}}{E_{1}+E_{2}}, \quad|\varepsilon| \ll 1, \quad|\gamma| \ll 1 .
$$


Для твердых тел с трещинами $E_{2}>E_{1}$, но для других материалов может быть и наоборот, $E_{2}<E_{1}$. Заметим, что нелинейность уравнения состояния (1) описывается неаналитической функцией $f(\varepsilon)=\gamma|\varepsilon|-$ непрерывной, но негладкой и недифференцируемой в точке $\varepsilon=0$. Из уравнения (1) следует, что одиночные положительные $(\varepsilon \geq 0)$ и отрицательные $(\varepsilon \leq 0)$ волновые возмущения в такой среде распространяются со скоростями $C_{+}$и $C_{-}$соответственно, причем

$$
C_{ \pm}=\left(E_{1,2} / \rho\right)^{1 / 2}=\sqrt{(E / \rho)(1 \mp \gamma)},
$$

$\rho-$ плотность среды. Мы не будем учитывать геометрическую нелинейность уравнений движения (по сравнению с нелинейностью уравнения состояния), полагая, что $|\varepsilon| \ll|\gamma|[5]$. В этом приближении $\rho=$ const, при этом уравнения теории упругости в лагранжевых и эйлеровых координатах совпадают [5].

Получим волновое уравнение для продольной деформации стержня, обладающего разномодульной нелинейностью, с учетом его линейной диссипации и геометрической дисперсии. Подставляя уравнение состояния (1) в уравнение движения $\rho U_{t t}=\sigma_{x}(\varepsilon)$ [13], и, учитывая линейную диссипацию [13] и геометрическую дисперсию фазовой скорости продольных (вдоль оси $x$ ) упругих волн в стержне конечного диаметра $[14,15]$, получим одномерное квазилинейное волновое уравнение для смещения и деформации

$$
U_{t t}-C_{0}^{2} \varepsilon_{x}=-\gamma C_{0}^{2}[|\varepsilon|]_{x}+\alpha \varepsilon_{t x}+v^{2} r_{0}^{2}\left[U_{t t}-C_{s h}^{2} U_{x x}\right]_{x x},
$$

где $U(x, t)$ и $\varepsilon(x, t)=\partial U(x, t) / \partial x-$ продольные смещение и деформация, $C_{0}=(E / \rho)^{1 / 2}, C_{s h}=(\mu / \rho)^{1 / 2}=$ $=[E / 2(1+v) \rho]^{1 / 2}=C_{0} /[2(1+v)]^{1 / 2}-$ скорость сдвиговой волны, $\mu=E / 2(1+v)$ - модуль сдвига, $v-$ коэффициент Пуассона, $\alpha$ - коэффициент диссипации, $r_{0}=R / \sqrt{2}, R-$ радиус стержня, $R<\Lambda / 2, \Lambda$ - длина волны. Дифференцируя уравнение (2) по $x$, получим волновое уравнение для деформации $\varepsilon$ :

$$
\varepsilon_{t t}-C_{0}^{2} \varepsilon_{x x}=-\gamma C_{0}^{2}[|\varepsilon|]_{x x}+\alpha \varepsilon_{t x x}+\nu^{2} r_{0}^{2}\left[\varepsilon_{t t}-C_{s h}^{2} \varepsilon_{x x}\right]_{x x} .
$$

Это уравнение аналогично уравнению с двумя дисперсиями [15]; отличие заключается в замене нелинейного квадратичного слагаемого на нелинейное разномодульное $\left(-\gamma C_{0}^{2}[|\varepsilon|]_{x x}\right)$ и наличии слагаемого $\alpha \varepsilon_{t x x}$, учитывающего линейную диссипацию волны.

Полагая нелинейное, диссипативное и дисперсионное слагаемые в правой части уравнения (3) малыми, упростим это уравнение, используя метод медленноменяющегося профиля $[14,16]$. Переходя в уравнении (3) к сопровождающей системе координат $\tau=t-x / C_{0}$, $x^{\prime}=x \geq 0$ и полагая в левой части уравнения (2)

$$
\begin{gathered}
\partial^{2} \varepsilon / \partial t^{2}=\partial^{2} \varepsilon / \partial \tau^{2}, \\
\partial^{2} \varepsilon / \partial x^{2} \approx\left(1 / C_{0}^{2}\right) \partial^{2} \varepsilon / \partial \tau^{2}-\left(2 / C_{0}\right) \partial^{2} \varepsilon / \partial x \partial \tau,
\end{gathered}
$$

а в правой -

$$
\begin{gathered}
\partial^{2}|\varepsilon| / \partial x^{2} \approx\left(1 / C_{0}^{2}\right) \partial^{2}|\varepsilon| / \partial \tau^{2}, \\
\partial^{3} \varepsilon / \partial t \partial x^{2} \approx\left(1 / C_{0}^{2}\right) \partial^{3} \varepsilon / \partial \tau^{3}, \\
\partial^{4} \varepsilon / \partial x^{4} \approx\left(1 / C_{0}^{4}\right) \partial^{4} \varepsilon / \partial \tau^{4}, \\
\partial^{4} \varepsilon / \partial x^{2} \partial t^{2} \approx\left(1 / C_{0}^{2}\right) \partial^{4} \varepsilon / \partial \tau^{4},
\end{gathered}
$$

получим эволюционное уравнение для волны деформации, бегущей в положительном направлении оси $x$ :

$$
\frac{\partial \varepsilon}{\partial x}=-\frac{\gamma}{2 C_{0}} \frac{\partial|\varepsilon|}{\partial \tau}+\frac{\alpha}{2 C_{0}^{3}} \frac{\partial^{2} \varepsilon}{\partial \tau^{2}}+\frac{v^{2} R^{2}(1+2 v)}{8 C_{0}^{3}(1+v)} \frac{\partial^{3} \varepsilon}{\partial \tau^{3}} .
$$

Отметим, что для бегущей волны имеет место соотношение $\varepsilon(x, \tau)=-V(x, \tau) / C_{0}, \quad$ где $V(x, \tau)=\partial U(x, \tau) / \partial \tau-$ скорость частиц среды.

Для получения и анализа решений уравнения (4) приведем его к безразмерному виду

$$
\frac{\partial e}{\partial z}=-\frac{\partial|e|}{\partial \theta}+\mu \frac{\partial^{2} e}{\partial \theta^{2}}+\delta \frac{\partial^{3} e}{\partial \theta^{3}},
$$

где $e=\varepsilon / \varepsilon_{0}, \theta=\omega \tau, z=\gamma \omega x / 2 C_{0}, \quad \varepsilon_{0}$ и $\omega-$ характерные амплитуда и частота волны, $\mu=\alpha \omega / \gamma C_{0}^{2}$, $\delta=v^{2} R^{2}(1+2 v) \omega^{2} / 4 \gamma(1+v) C_{0}^{2}$. Коэффициент $\mu-$ это коэффициент, обратный акустическому числу Рэйнольдса, а коэффициент $\delta=U r^{-1}-$ это обратный параметр Урселла $U r=4 \gamma(1+v) C_{0}^{2} / v^{2} R^{2}(1+2 v) \omega^{2}[14,16]$. Для разномодульной среды число Рэйнольдса и параметр Урселла не зависят от амплитуды волны $\varepsilon=\varepsilon(x, \tau)$, поэтому и решения уравнения (4) также не зависят от этой амплитуды, т.е. если функция $\varepsilon=\varepsilon(x, \tau)$ является решением уравнения (4), то и функция $\varepsilon=C \varepsilon(x, \tau)$, где $C=$ const $>0$, также является решением этого уравнения.

Волновое уравнение (5) - это аналог уравнения Кортевега-де Вриза-Бюргерса $[14,16]$ для среды с разномодульной нелинейностью. Квазилинейное уравнение (5) - это, по существу, два линейных дифференциальных уравнения третьего порядка: одно - для положительной части $\left[e_{+}(z, \theta) \geq 0\right]$ волны $e=e(z, \theta)$, другое - для отрицательной $\left[e_{-}(z, \theta) \leq 0\right]$. Положительная и отрицательная части волны непрерывно (вместе с производными $\partial e_{ \pm}(z, \theta) / \partial \theta$ и $\left.\partial^{2} e_{ \pm}(z, \theta) / \partial \theta^{2}\right)$ переходят друг в друга при $e_{ \pm}(z, \theta)=0$.

Из уравнения (5) следует, что эволюция волны в разномодульном стержне зависит от двух коэффициентов $\mu$ и $\delta$, причем в случае $\mu \ll 1$ и $|\delta| \ll 1$ получим сильнонелинейную волну, а в случае $\mu \gg 1$ или $|\delta| \gg 1-$ практически ее линейное распространение. Далее для определенности мы будем полагать, что $\gamma>0$ и, следовательно, $\delta>0, \mu>0$ и $z \geq 0$. 

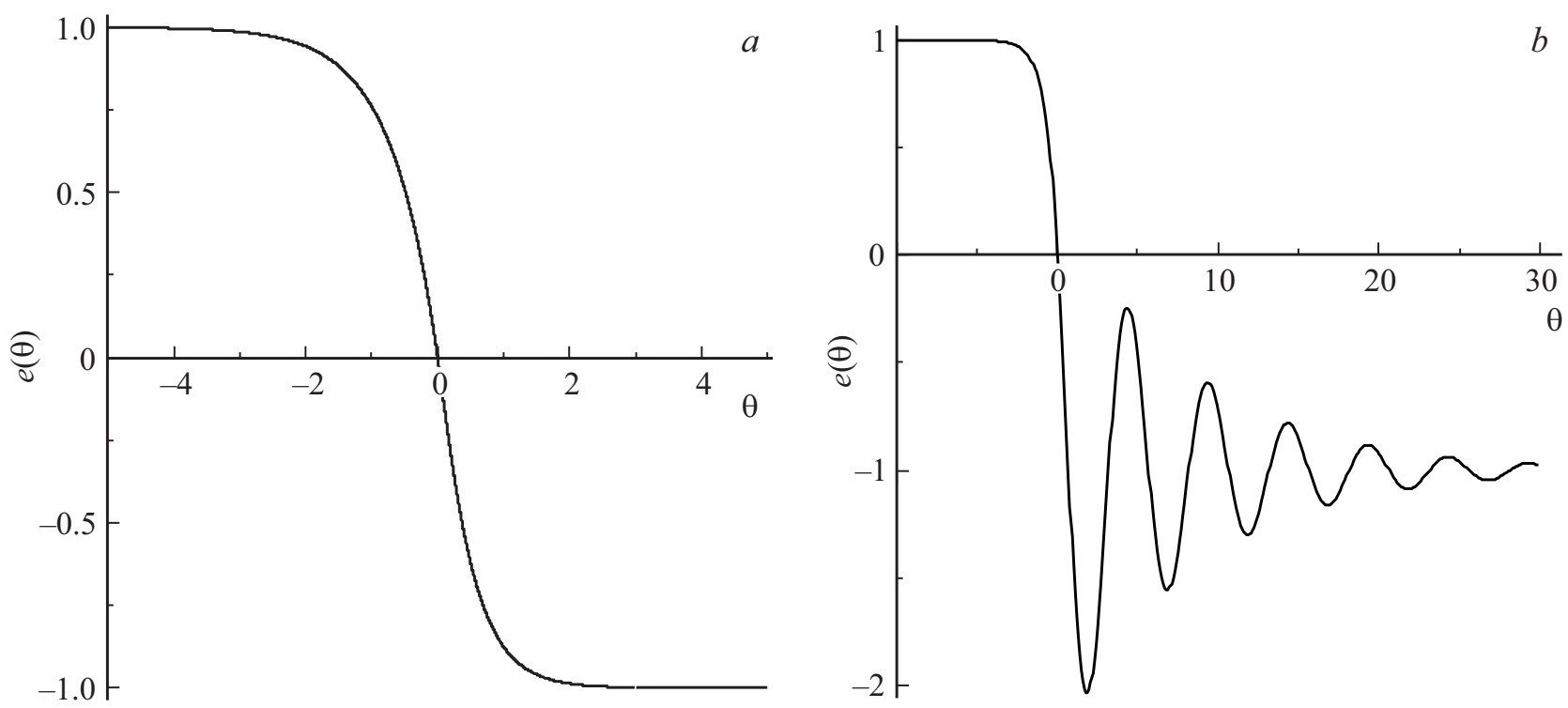

Рис. 1. Форма стационарной волны при $\delta=0.07, \mu=0.6, \mu^{2}>4 \delta(a)$; при $\delta=0.4, \mu=0.1, \mu^{2}<4 \delta(b)$.

\section{2. Стационарные волны}

Как и уравнения Бюргерса и Кортевега-де Вриза $[14,16]$, нелинейное уравнение (5) имеет частные точные решения, описывающие стационарные волны, не зависящие от координаты $z$, и распространяющиеся без изменения формы. Полагая в уравнении (5) $\partial e(z, \theta) / \partial z=0$, получаем уравнение для стационарной волны типа скачка-перехода (при $\gamma>0$ ) из состояния $e_{-}(\theta \rightarrow \infty)=-1$ в состояние $e_{+}(\theta \rightarrow-\infty)=1$ :

$$
\delta \frac{d^{2} e}{d \theta^{2}}+\mu \frac{d e}{d \theta}-|e|=-1 .
$$

(При $\gamma<0$ устойчивым будет обратный переход: из $e_{+}(\theta \rightarrow-\infty)=1$ в $e_{-}(\theta \rightarrow \infty)=-1$.) При $\mu^{2}>4 \delta$ решение уравнения (5) имеет вид плавного перехода ступеньки (рис. $1, a$ )

$$
\begin{gathered}
e_{+}(\theta \leq 0)=1-\exp \left(\lambda_{1}^{+} \theta\right) \geq 0 \\
e_{-}(\theta \geq 0)=-1+\frac{1}{2}\left(1+\frac{2 \mu-\sqrt{\mu^{2}+4 \delta}}{\sqrt{\mu^{2}-4 \delta}}\right) \exp \left(\lambda_{3}^{-} \theta\right) \\
+\frac{1}{2}\left(1-\frac{2 \mu-\sqrt{\mu^{2}+4 \delta}}{\sqrt{\mu^{2}-4 \delta}}\right) \exp \left(\lambda_{4}^{-} \theta\right) \leq 0
\end{gathered}
$$

где

$$
\lambda_{1,2}^{+}=\left[-\mu \pm \sqrt{\mu^{2}+4 \delta}\right] / 2 \delta, \quad \lambda_{1}^{+} \geq 0, \quad \lambda_{2}^{+}<0,
$$

$\lambda_{3,4}^{-}=\left[-\mu \pm \sqrt{\mu^{2}-4 \delta}\right] / 2 \delta \leq 0, \quad \lambda_{3}^{-} \leq 0, \quad \lambda_{4}^{-}<0$.

При $\mu^{2}<4 \delta$ и выполнении условия

$$
\begin{aligned}
& \ln \left(\frac{2 \sqrt{2 \delta}}{\mu+\sqrt{4 \delta+\mu^{2}}}\right) \leq \frac{\mu}{\sqrt{4 \delta-\mu^{2}}} \\
& \quad \times\left(2 \pi-\operatorname{arctg} \frac{\sqrt{4 \delta-\mu^{2}}}{\mu+\sqrt{4 \delta+\mu^{2}}}\right),
\end{aligned}
$$

т.е. при $\frac{1}{4}<\frac{\delta}{\mu^{2}}<88.81$, решение уравнения (6) имеет вид (рис. $1, b$ )

$$
\begin{gathered}
e_{+}(\theta \leq 0)=1-\exp \left(\lambda_{1}^{+} \theta\right) \geq 0, \\
e_{-}(\theta \geq 0)=-1+\frac{2 \mu-\sqrt{\mu^{2}+4 \delta}}{\sqrt{\left|\mu^{2}-4 \delta\right|}} \exp \left(-\frac{\mu \theta}{2 \delta}\right) \\
\\
\times \sin \left(\frac{\sqrt{\left|\mu^{2}-4 \delta\right|}}{2 \delta} \theta\right)+\exp \left(-\frac{\mu \theta}{2 \delta}\right) \\
\\
\times \cos \left(\frac{\sqrt{\left|\mu^{2}-4 \delta\right|}}{2 \delta} \theta\right) \leq 0 .
\end{gathered}
$$

Здесь отрицательная часть волны $e=e(\theta)$ содержит затухающие колебания с частотой $p=\sqrt{\left|\mu^{2}-4 \delta\right|} / 2 \delta$.

При $\mu^{2}=4 \delta$ решение (8) имеет вид

$$
\begin{gathered}
e_{+}(\theta \leq 0)=1-\exp \left(\frac{2(\sqrt{2}-1) \theta}{\mu}\right) \geq 0, \\
e_{-}(\theta \geq 0)=-1+\left(1+\frac{2(2-\sqrt{2}) \theta}{\mu}\right) \exp \left(-\frac{2 \theta}{\mu}\right) \leq 0 .
\end{gathered}
$$

Форма такой волны подобна изображенной на рис. $1, a$.

Из-за неаналитичности разномодульной нелинейности уравнения (5), его решения для стационарных волн получились также неаналитическими: положительная и отрицательная части волны $e=e(\theta)$ описываются разными функциями $e_{ \pm}(\theta)$, при этом в точке $\theta_{0}=0$ терпит разрыв третья производная $d^{3} e_{ \pm}\left(\theta_{0}\right) / d \theta^{3}$. Здесь, как и в среде с квадратичной упругой нелинейностью $[14,16]$, устойчивость стационарных волн (7)-(9) обеспечивается балансом эффектов нелинейности, диссипации и дисперсии: разномодульная нелинейность увеличивает крутизну фронта, а диссипация и дисперсия стремятся ее уменьшить. 


\section{3. Самоподобные периодические волны и импульсные возмущения}

Для нахождения автомодельных (или самоподобных) решений [7-10,16] уравнения (5) воспользуемся методом разделения переменных, полагая, что

$$
e(z, \theta)=Z(z) \Psi(\theta), \quad Z(z=0)=1, \quad Z(z)>0 .
$$

Решение (10) описывает затухающую самоподобную волну, не меняющую своей формы $\Psi=\Psi(\theta)$ при распространении [7-10]. Примерами самоподобных волн являются рассмотренные выше стационарные волны и солитоны [14-16] в средах с квадратичной нелинейностью, линейной диссипацией и дисперсией соответственно и гармонические волны в линейных средах.

Подставляя (10) в (5), получаем

$$
\begin{aligned}
\frac{1}{Z(z)} \frac{d Z(z)}{d z}= & \frac{1}{\Psi(\theta)}\left(\delta \frac{d^{3} \Psi(\theta)}{d \theta^{3}}+\mu \frac{d^{2} \Psi(\theta)}{d \theta^{2}}\right. \\
& \left.-\frac{d|\Psi(\theta)|}{d \theta}\right)=-m,
\end{aligned}
$$

где $m=$ const. Из физических соображений - волна не должна быть нарастающей по координате $z>0$, следует, что $m \geq 0$, при этом $Z(z)=\exp (-m z)$. Значение параметра зависит от $\delta$ и $\mu$ и определяется граничным условием для излучаемой самоподобной волны $e(z=0, \theta)=\Psi(\theta)$, удовлетворяющей линейным уравнениям для положительной $\Psi_{+}(\theta) \geq 0$ и отрицательной $\Psi_{-}(\theta) \leq 0$ частям функции $\Psi(\theta)$ :

$$
\delta \frac{d^{3} \Psi_{ \pm}(\theta)}{d \theta^{3}}+\mu \frac{d^{2} \Psi_{ \pm}(\theta)}{d \theta^{2}} \mp \frac{d \Psi_{ \pm}(\theta)}{d \theta}+m \Psi_{ \pm}(\theta)=0 .
$$

Из уравнений (12) получаем характеристическое уравнение

$$
\delta\left(\lambda^{ \pm}\right)^{3}+\mu\left(\lambda^{ \pm}\right)^{2} \mp\left(\lambda^{ \pm}\right)+m=0,
$$

где характеристические числа $\lambda^{ \pm}$соответствуют положительным $\Psi_{+}(\theta) \geq 0$ и отрицательным $\Psi_{-}(\theta) \leq 0$ частям функции $\Psi(\theta)$.

Корни $\lambda_{1,2,3}^{ \pm}$кубического уравнения (13) определяются формулой Кардано [17]:

$$
\begin{gathered}
\lambda_{1}^{ \pm}=A_{ \pm}+B_{ \pm}-\frac{\mu}{3 \delta} \\
\lambda_{2,3}^{ \pm}=-\frac{A_{ \pm}+B_{ \pm}}{2} \pm i \frac{\sqrt{3}\left(A_{ \pm}-B_{ \pm}\right)}{2}-\frac{\mu}{3 \delta},
\end{gathered}
$$

где

$$
\begin{gathered}
A_{ \pm}=\left(-\frac{q_{ \pm}}{2}+\sqrt{Q_{ \pm}}\right)^{1 / 3}, B_{ \pm}=\left(-\frac{q_{ \pm}}{2}-\sqrt{Q_{ \pm}}\right)^{1 / 3} \\
Q_{ \pm}=\left(\frac{p_{ \pm}}{3}\right)^{3}+\left(\frac{p_{ \pm}}{2}\right)^{2}
\end{gathered}
$$

$$
p_{ \pm}=-\frac{1}{3}\left(\frac{\mu}{\delta}\right)^{2} \mp \frac{1}{\delta}, q_{ \pm}=2\left(\frac{\mu}{3 \delta}\right)^{3} \pm \frac{\mu}{3 \delta^{2}}+\frac{m}{\delta} .
$$

В зависимости от параметров $\delta, \mu$ и $m$, возможны различные варианты решений (14). В первом варианте: $\lambda_{1}^{+}$- действительное число, а $\lambda_{1,2}^{+}-$комплексносопряженные числа, и втором: все $\lambda_{1,2,3}^{+}-$действительные числа. Такие же варианты имеют место и для корней $\lambda_{1,2,3}^{-}$. Таким образом, здесь возможны различные решения уравнения (12) как в виде периодических волн, так и импульсных возмущений. Эти решения определяются следующими выражениями:

$$
\begin{aligned}
& \Psi_{+}(\theta)=A_{1}^{+} \exp \left(\lambda_{1}^{+} \theta\right)+A_{2}^{+} \exp \left(\lambda_{2}^{+} \theta\right)+A_{3}^{+} \exp \left(\lambda_{3}^{+} \theta\right)+c . c . \\
& \Psi_{-}(\theta)=A_{1}^{-} \exp \left(\lambda_{1}^{-} \theta\right)+A_{2}^{-} \exp \left(\lambda_{2}^{-} \theta\right)+A_{3}^{-} \exp \left(\lambda_{3}^{-} \theta\right)+c . c .,
\end{aligned}
$$

где коэффициенты $A_{1,2,3}^{ \pm}$и параметр $m$ зависят от вида волны (периодической или импульсной); они находятся из условий непрерывности функций $\Psi_{+}(\theta) \geq 0$, $\Psi_{-}(\theta) \leq 0$ и их производных $d \Psi_{ \pm}(\theta) / d \theta, d^{2} \Psi_{ \pm}(\theta) / d \theta^{2}$ в точках $\theta=\theta_{n}$, в которых $\Psi_{ \pm}\left(\theta_{n}\right)=0$ :

$$
\begin{gathered}
\Psi_{+}\left[n\left(\theta_{1}+\theta_{2}\right)\right]=\Psi_{-}\left[n\left(\theta_{1}+\theta_{2}\right)\right]=0, \\
\Psi_{+}\left[n\left(\theta_{1}+\theta_{2}\right)+\theta_{2}\right]=\Psi_{-}\left[n\left(\theta_{1}+\theta_{2}\right)+\theta_{2}\right]=0, \\
\frac{d \Psi_{+}\left[n\left(\theta_{1}+\theta_{2}\right)\right]}{d \theta}=\frac{d \Psi_{-}\left[n\left(\theta_{1}+\theta_{2}\right)\right]}{d \theta}, \\
\frac{d \Psi_{+}\left[n\left(\theta_{1}+\theta_{2}\right)+\theta_{2}\right]}{d \theta}=\frac{d \Psi_{-}\left[n\left(\theta_{1}+\theta_{2}\right)+\theta_{2}\right]}{d \theta}, \\
\frac{d^{2} \Psi_{+}\left[n\left(\theta_{1}+\theta_{2}\right)\right]}{d \theta^{2}}=\frac{d^{2} \Psi_{-}\left[n\left(\theta_{1}+\theta_{2}\right)\right]}{d \theta^{2}}, \\
\frac{d^{2} \Psi_{+}\left[n\left(\theta_{1}+\theta_{2}\right)+\theta_{2}\right]}{d \theta^{2}}=\frac{d^{2} \Psi_{-}\left[n\left(\theta_{1}+\theta_{2}\right)+\theta_{2}\right]}{d \theta^{2}},
\end{gathered}
$$

где $\theta_{1,2}$ - длительности функций $\Psi_{ \pm}(\theta), \theta_{1}+\theta_{2}-$ период функции $\Psi(\theta), n=0, \pm 1, \pm 2, \ldots$ Уравнения (16) представляют собой систему из восьми уравнений с восемью неизвестными (один из коэффициентов $A_{1,2,3}^{ \pm}$ для определенности, например $A_{1}^{+}$, можно положить равным единице), так что коэффициенты $A_{2,3}^{+}, A_{1,2,3}^{-}, \theta_{1,2}$ и параметр $m$ (при заданных $\delta$ и $\mu$ ) определяются однозначно. В общем виде, однако, получить аналитические выражения для $A_{2,3}^{+}, A_{1,2,3}^{-}, \theta_{1,2}$ и $m$ довольно сложно, поэтому далее мы рассмотрим несколько характерных частных случаев.

Если $\lambda_{1}^{ \pm}$- действительные числа, а $\lambda_{2,3}^{ \pm}-$ комплексно-сопряженные, то получим периодическую волну, при этом из (15) получаем выражения для $\Psi_{ \pm}(\theta)$ в более удобном виде (для одного периода волны):

$$
\begin{gathered}
\Psi_{+}(\theta)=-\exp \left(\lambda_{1}^{+} \theta\right) \sin \theta^{+}+\exp \left(\operatorname{Re} \lambda_{2}^{+} \theta\right) \\
\times \sin \left(\operatorname{Im} \lambda_{2}^{+} \theta+\theta^{+}\right) \geq 0, \\
\Psi_{-}(\theta)=-C^{-} \exp \left(\lambda_{1}^{-} \theta\right) \sin \theta^{-}+C^{-} \exp \left(\operatorname{Re} \lambda_{2}^{-} \theta\right) \\
\times \sin \left(\operatorname{Im} \lambda_{2}^{-} \theta+\theta^{-}\right) \leq 0,
\end{gathered}
$$$$
\text { где } C^{-}, \theta^{+}, \theta^{-} \text {и } m \text { определяются из условий }(16) \text {. }
$$ 

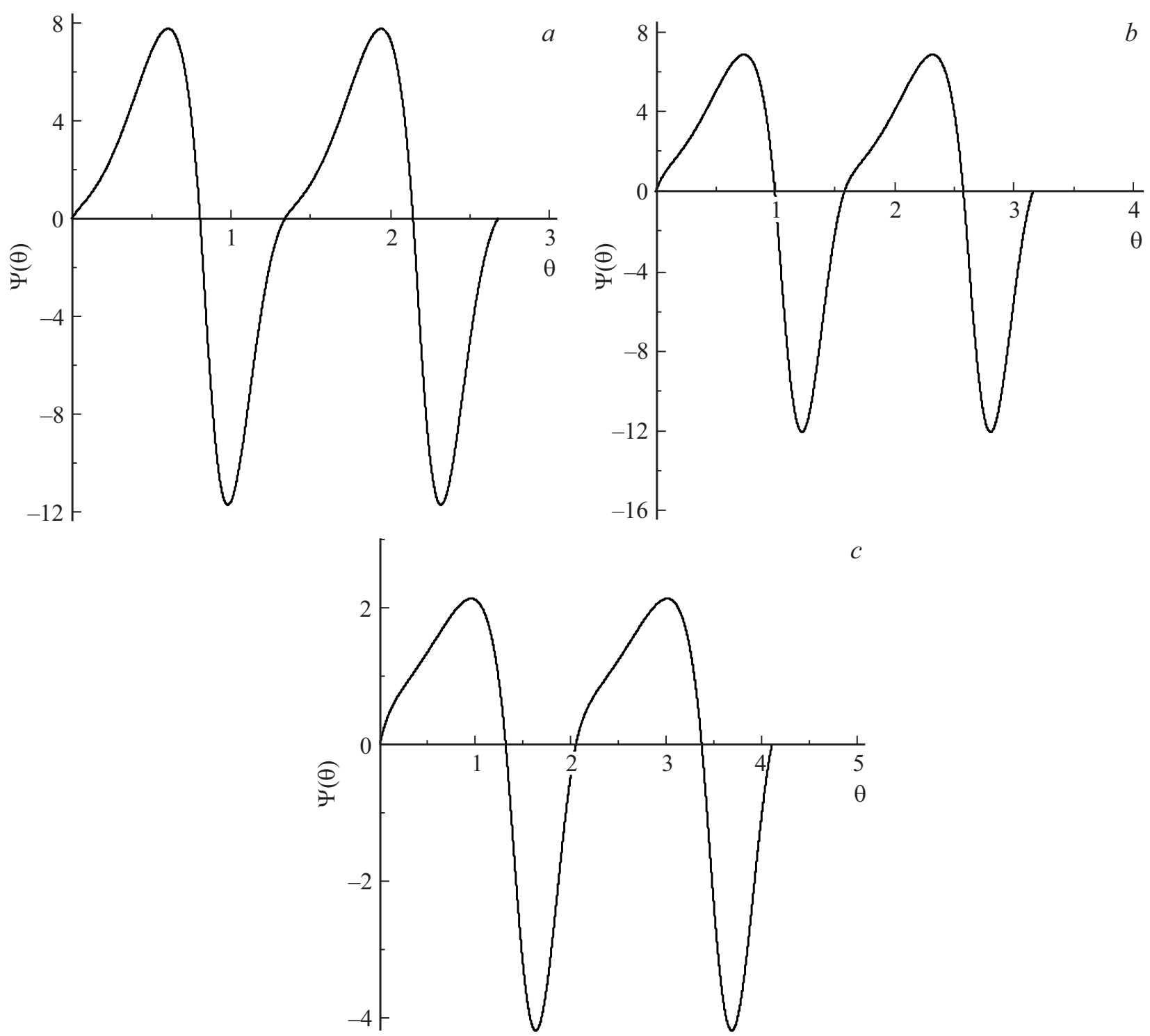

Рис. 2. Форма самоподобной периодической волны при $\delta=0.005, \mu=0.1, m=4.168, \lambda_{1}^{+}=-28.155, \lambda_{2,3}^{+}=4.078 \pm 3.603 i$, $\lambda_{1}^{-}=-8.024, \quad \lambda_{2,3}^{-}=-5.988 \pm 8.249 i, \quad C^{-}=-1.327, \quad \eta^{+}=0.244, \quad \eta^{-}=3.425, \quad \eta_{1}=0.804, \quad \eta_{2}=0.533 \quad(a) ; \quad$ при $\delta=0.01$, $\mu=0.1, \quad m=2.831, \quad \lambda_{1}^{+}=-16.906, \quad \lambda_{2,3}^{+}=3.453 \pm 2.197 i, \quad \lambda_{1}^{-}=-3.691, \quad \lambda_{2,3}^{-}=-3.154 \pm 8.171 i, \quad C^{-}=-2.611, \quad \eta^{+}=0.952$, $\eta^{-}=3.789, \eta_{1}=0.996, \eta_{2}=0.582(b) ;$ при $\delta=0.02, \mu=0.1, m=1.627, \lambda_{1}^{+}=-10.5, \lambda_{2,3}^{+}=2.75 \pm 0.431 i, \quad \lambda_{1}^{-}=-1.841$, $\lambda_{2,3}^{-}=-1.579 \pm 6.457 i, C^{-}=-1.328, \theta^{+}=2.574, \theta^{-}=3.846, \theta_{1}=1.316, \theta_{2}=0.736(c)$.

На рис. 2 приведены некоторые характерные формы самоподобных периодических волн, построенных при различных параметрах $\delta$ и $\mu$.

При условии $\Psi_{ \pm}(\theta \rightarrow \mp \infty) \rightarrow \pm 0$ получим самоподобные импульсные возмущения. В этом случае, если $\lambda_{1,2,3}^{ \pm}$действительные числа и $0<\lambda_{1}^{+}<\lambda_{2}^{+}$, то из выражений (15) имеем

$$
\begin{aligned}
\Psi^{+}(\theta \leq 0) & =\exp \left(\lambda_{1}^{+} \theta\right)-\exp \left(\lambda_{2}^{+} \theta\right) \geq 0, \\
\Psi^{-}(\theta \geq 0)= & C_{1} \exp \left(\lambda_{1}^{-} \theta\right)+C_{2} \exp \left(\lambda_{2}^{-} \theta\right) \\
& +C_{3} \exp \left(\lambda_{3}^{-} \theta\right) \leq 0,
\end{aligned}
$$

где

$$
\begin{aligned}
& C_{1}=\frac{\left(\lambda_{1}^{+}-\lambda_{2}^{+}\right)\left(\lambda_{1}^{+}+\lambda_{2}^{+}-\lambda_{3}^{-}-\lambda_{2}^{-}\right)}{\left(\lambda_{1}^{-}-\lambda_{2}^{-}\right)\left(\lambda_{1}^{-}-\lambda_{3}^{-}\right)}, \\
& C_{2}=\frac{\left(\lambda_{1}^{+}-\lambda_{2}^{+}\right)\left(\lambda_{1}^{+}+\lambda_{2}^{+}-\lambda_{3}^{-}-\lambda_{1}^{-}\right)}{\left(\lambda_{2}^{-}-\lambda_{1}^{-}\right)\left(\lambda_{2}^{-}-\lambda_{3}^{-}\right)}, \\
& C_{3}=\frac{\left(\lambda_{1}^{+}-\lambda_{2}^{+}\right)\left(\lambda_{1}^{+}+\lambda_{2}^{+}-\lambda_{2}^{-}-\lambda_{1}^{-}\right)}{\left(\lambda_{3}^{-}-\lambda_{1}^{-}\right)\left(\lambda_{3}^{-}-\lambda_{2}^{-}\right)} .
\end{aligned}
$$

Форма импульсов (18) приведена на рис. $3, a$.

Если же $\lambda_{1,2}^{+}>0, \lambda_{1}^{-}<0-$ действительные числа, а $\lambda_{2,3}^{-}-$комплексно-сопряженные, $\lambda_{2}^{+}>\lambda_{1}^{+}$, 

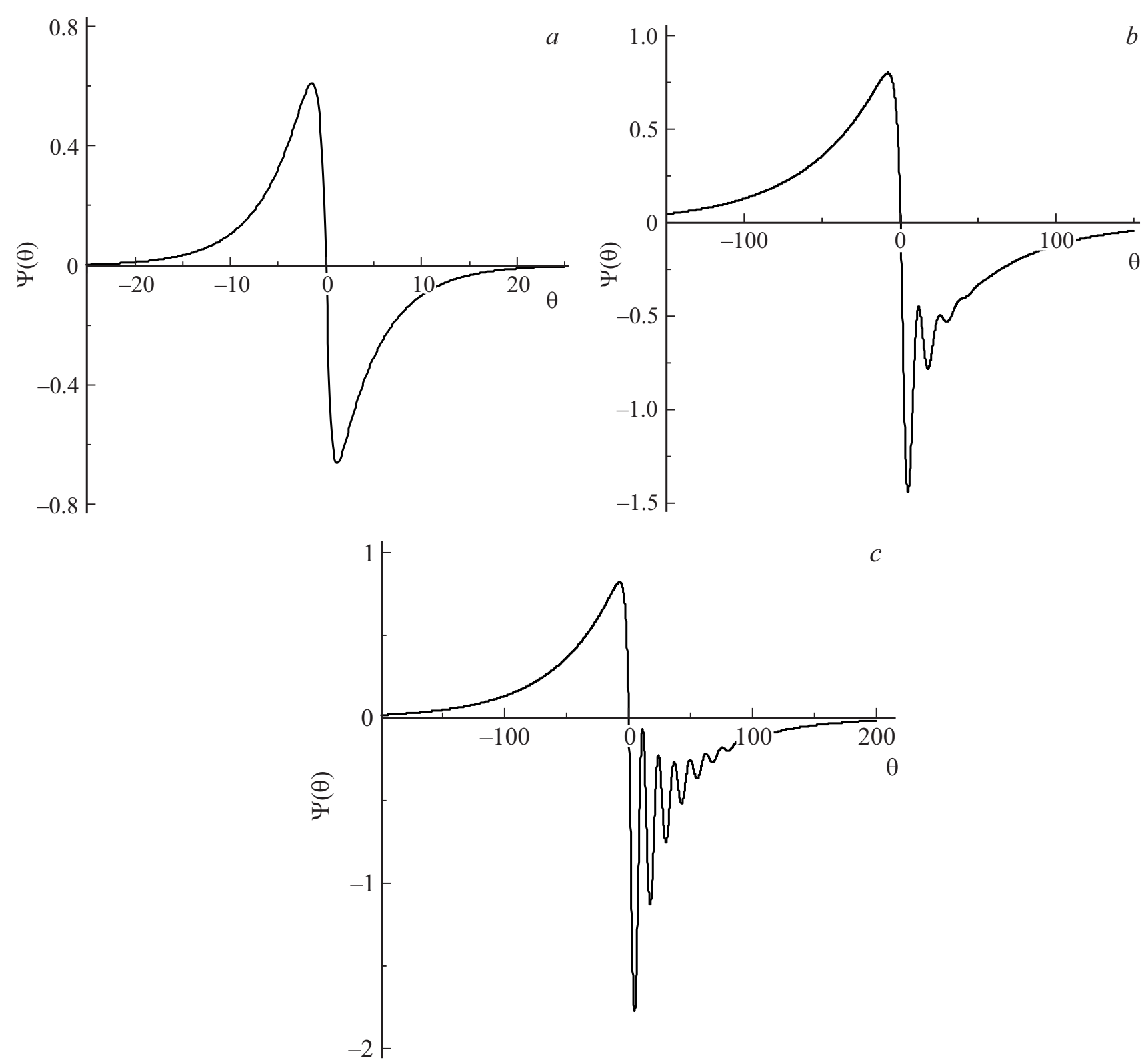

Рис. 3. Форма самоподобного импульса при $\delta=0.05, \mu=0.5, m=0.2, \lambda_{1}^{+}=0.226, \lambda_{2}^{+}=1.507, \lambda_{1}^{-}=-0.225, \lambda_{2}^{-}=-2.421$, $\lambda_{3}^{-}=-7.355, C_{1}^{-}=-0.942, C_{2}^{-}=1.101, C_{3}^{-}=-0.159(a)$; при $\delta=4, \mu=1, m=0.02, \lambda_{1}^{+}=0.020, \lambda_{2}^{+}=0.377, \lambda_{1}^{-}=-0.020$, $\lambda_{2}^{-}=-0.115+0.482 i, \lambda_{3}^{-}=-0.115-0.482 i, C_{1}^{-}=-1.084, \theta^{-}=-1.029 \quad(b) ;$ при $\delta=4, \mu=0.5, m=0.02, \lambda_{1}^{+}=0.020$, $\lambda_{2}^{+}=0.430, \lambda_{1}^{-}=-0.020, \lambda_{2}^{-}=-0.052+0.495 i, \lambda_{3}^{-}=-0.052-0.495 i, C_{1}^{-}=-1.200, \theta^{-}=-0.877$ (c).

$\operatorname{Re} \lambda_{2}^{-}<\lambda_{1}^{-}<0$, то из выражений (15) имеем

$$
\begin{gathered}
\Psi_{+}(\theta \leq 0)=\exp \left(\lambda_{1}^{+} \theta\right)-\exp \left(\lambda_{2}^{+} \theta\right) \geq 0 \\
\Psi_{-}(\theta \geq 0)=-C_{1}^{-} \exp \left(\lambda_{1}^{-} \theta\right) \sin \left(\theta^{-}\right)+C_{1}^{-} \exp \left(\operatorname{Re} \lambda_{2}^{-} \theta\right) \\
\times \sin \left(\operatorname{Im} \lambda_{2}^{-} \theta+\theta^{-}\right) \leq 0,
\end{gathered}
$$

где $C_{1}^{-}$и $\theta^{-}$также находятся из условий непрерывности функций $\Psi_{+}(\theta) \geq 0$ и $\Psi_{-}(\theta) \leq 0$ и их производных $d \Psi_{ \pm}(\theta) / d \theta, \quad d^{2} \Psi_{ \pm}(\theta) / d \theta^{2}$ в точке $\theta=0$, в которой $\Psi_{ \pm}(\theta=0)=0$.

Форма таких импульсов показана на рис. 3. Здесь, как и в стационарной волне, отрицательная часть $\Psi_{-}(\theta) \leq 0$ импульсных возмущений содержит затухающие колебания.

Из рис. 2 и 3 видно, что в зависимости от параметров $\delta, \mu$, и $m$ формы самоподобных периодических волн и импульсных возмущений довольно разнообразны, при этом профили, амплитуды и длительности положительной и отрицательной частей для каждой такой волны различны. Отметим, что при возбуждении и распространении в разномодульной среде с диссипацией (или дисперсией) первоначально гармонической волны или одиночных разнополярных импульсов, их формы (по мере распространения) будут асимптотически приближаться 
к формам описанных в настоящей работе самоподобных периодических волн и импульсных возмущений [7-10].

\section{Заключение}

Исследовано распространение продольных упругих волн в стержне с разномодульной нелинейностью, линейной диссипацией и геометрической дисперсией фазовой скорости. Показано, что волны в таком стержне описываются уравнением, аналогичным уравнению Кортевега-де Вриза-Бюргерса, для среды с квадратичной нелинейностью $[14,16]$. Получены точные аналитические и численные решения для профилей разнополярных стационарных волн и самоподобных периодических волн и импульсных возмущений, распространяющихся с затуханием, но без изменения формы. Результаты проведенных исследований представляют интерес для развития теории волновых процессов в средах с неаналитической нелинейностью; они также могут быть использованы и для создания нелинейных методов акустической диагностики структурно-неоднородных сред и конструкционных материалов, содержащих трещины. Основой таких методов является выявление закономерностей нелинейных процессов распространения и взаимодействия первичных гармонических волн и эффектов генерации вторичных волн на комбинационных частотах, отсутствующих в спектре первичных волн.

\section{Финансирование работы}

Работа выполнена в рамках государственного задания ИПФ РАН по теме № 0035-2019-0009.

\section{Конфликт интересов}

Авторы заявляют, что у них нет конфликта интересов.

\section{Список литературы}

[1] Амбариумян С.A. Разномодульная теория упругости. М.: Наука, 1982. 359 c.

[2] Николаев А.В. // Изв. АН СССР. Физика Земли. 1979. № 1. C. $72-77$.

[3] Benveniste Y. // Intern. J. Engineer. Sci. 1980. Vol. 18. N 6. P. $815-827$.

[4] Маслов В.П., Мосолов П.П. // ПМТФ. 1985. Т. 49. № 3. C. $419-437$.

[5] Назаров В.Е., Островский Л.А. // Акуст. журн. 1990. Т. 36. № 1. C. $106-110$.

[6] Gavrilov S.N., Herman G.C. // J. Sound and Vibration. 2012. Vol. 331. P. 4464-4480.

[7] Radostin A.V., Nazarov V.E., Kiyashko S.B. // Wave Motion. 2013. Vol. 50. N 2. P. 191-196.

[8] Назаров В.Е., Кияшко С.В., Радостин А.В. // Изв. вузов. Радиофизика. 2015. Т. 58. № 2. С. 134-141.

[9] Назаров В.Е., Кияшко С.В., Радостин А.В. // Изв. вузов. Радиофизика. 2015. Т. 58. № 10. С. 811-820.
[10] Назаров В.Е., Кияшко С.В., Радостин А.В. // Изв. вузов. Радиофизика. 2016. Т. 59. № 3. С. 275-285.

[11] Rudenko O.V. // Modular Solitons. Doklady Mathematics. 2016. Vol. 94. P. 708-711.

[12] Nazarov V.E., Kiyashko S.B., Radostin A.V. // Wave Motion. 2017. Vol. 75. P. 72-76.

[13] Ландау Л.Д., Либшии, Е.M. Теория упругости. М.: Наука, 1965. $204 \mathrm{c}$.

[14] Наугольных К.А., Островский Л.А. Нелинейные процессы в акустике. М.: Наука, 1990. 240 с.

[15] Порубов А.В. Локализация нелинейных волн деформации. М.: Физматлит, 2009. 208 с.

[16] Руденко О.В., Солуян С.И. Теоретические основы нелинейной акустики. М.: Наука, 1975. 288 с.

[17] Корн Г., Корн Т. Справочник по математике. М.: Наука, 1970. $720 \mathrm{c}$. 